Otterbein University

Digital Commons @ Otterbein

Physics Faculty Scholarship

Physics

7-15-1997

\title{
Vacuum Structure of Two-Dimensional Gauge Theories on the Light Front
}

\author{
Gary McCartor \\ Southern Methodist University \\ David G. Robertson \\ Otterbein University \\ Stephen S. Pinksy \\ Ohio State University
}

Follow this and additional works at: https://digitalcommons.otterbein.edu/phys_fac

Part of the Physics Commons

\section{Repository Citation}

McCartor, Gary; Robertson, David G.; and Pinksy, Stephen S., "Vacuum Structure of Two-Dimensional Gauge Theories on the Light Front" (1997). Physics Faculty Scholarship. 14.

https://digitalcommons.otterbein.edu/phys_fac/14

This Article is brought to you for free and open access by the Physics at Digital Commons @ Otterbein. It has been accepted for inclusion in Physics Faculty Scholarship by an authorized administrator of Digital Commons @ Otterbein. For more information, please contact digitalcommons07@otterbein.edu. 


\title{
Vacuum structure of two-dimensional gauge theories on the light front
}

\author{
Gary McCartor \\ Department of Physics, Southern Methodist University, Dallas, Texas 75275 \\ David G. Robertson and Stephen S. Pinsky \\ Department of Physics, The Ohio State University, Columbus, Ohio 43210
}

(Received 9 December 1996)

\begin{abstract}
We discuss the problem of vacuum structure in light-front field theory in the context of $(1+1)$-dimensional gauge theories. We begin by reviewing the known light-front solution of the Schwinger model, highlighting the issues that are relevant for reproducing the $\theta$ structure of the vacuum. The most important of these are the need to introduce degrees of freedom initialized on two different null planes, the proper incorporation of gauge field zero modes when periodicity conditions are used to regulate the infrared, and the importance of carefully regulating singular operator products in a gauge-invariant way. We then consider SU(2) Yang-Mills theory in $1+1$ dimensions coupled to massless adjoint fermions. With all fields in the adjoint representation the gauge group is actually $\mathrm{SU}(2) / Z_{2}$, which possesses nontrivial topology. In particular, there are two topological sectors and the physical vacuum state has a structure analogous to a $\theta$ vacuum. We formulate the model using periodicity conditions in $x^{ \pm}$for infrared regulation, and consider a solution in which the gauge field zero mode is treated as a constrained operator. We obtain the expected $Z_{2}$ vacuum structure, and verify that the discrete vacuum angle which enters has no effect on the spectrum of the theory. We then calculate the chiral condensate, which is sensitive to the vacuum structure. The result is nonzero, but inversely proportional to the periodicity length, a situation which is familiar from the Schwinger model. The origin of this behavior is discussed. [S0556-2821(97)00414-1]
\end{abstract}

PACS number(s): 11.10.Kk, 11.10.Ef, 11.15.Tk

\section{INTRODUCTION}

Light-front quantization [1] has recently emerged as a potentially powerful tool for the nonperturbative study of quantum field theories [2]. The main advantage of this approach is the apparent simplicity of the vacuum state, which leads to major simplifications in the solution of the Hamiltonian eigenvalue problem. Indeed, naive arguments suggest that the physical vacuum is trivial on the light front. In many theories of interest, however, the structure of the vacuum plays an important physical role, giving rise to, e.g., spontaneous symmetry breaking, confinement, vacuum angles, etc. It is therefore necessary to understand how these phenomena can occur in light-front field theory.

These issues have recently been discussed in a variety of contexts. If one regulates the infrared by imposing periodic or antiperiodic boundary conditions on some finite interval in $x^{-}[3,4]$, then any nontrivial vacuum structure must be connected with the $k^{+}=0$ Fourier modes of the fields. ${ }^{1}$ Studies of model field theories have shown that certain aspects of vacuum physics can in fact be reproduced by a careful treatment of the field zero modes in this framework. For example, it has been shown that solutions of the zero-mode constraint equation in $\phi_{1+1}^{4}$ theory [3] exhibit spontaneous symmetry breaking [5-9]. In addition, certain topological features of pure Yang-Mills theories in $1+1$ dimensions have been successfully reproduced [10].

The focus of the present work is on structure of the $\theta$-vacuum type in gauge theories. This has been discussed in

${ }^{1}$ This follows from simple kinematical considerations. detail for the Schwinger model in [11] (see also [12] for a discussion in the bosonized context), where it was shown that in order to obtain a theory that is isomorphic to the usual equal-time theory it is necessary to go beyond the conventional light-front approach. The main complication is the need to introduce degrees of freedom initialized along a second null plane, specifically a surface of constant $x^{-}$. In addition, it is important to properly treat the gauge field zero modes and to carefully define singular operator products in a gauge-invariant way.

Non-Abelian realizations of this sort of vacuum structure are difficult to find in $1+1$ dimensions, however, due to the fact that $\Pi_{1}[\operatorname{SU}(N)]$ is trivial. A model which does exhibit a sort of $\theta$ vacuum is Yang-Mills theory coupled to fermions in the adjoint representation. Since all fields transform according to the adjoint representation, gauge transformations that differ by an element in the center of the gauge group represent the same transformation and so should be identified. Thus the gauge group is actually $\mathrm{SU}(N) / Z_{N}$, which has nontrivial topology: $\Pi_{1}\left[\mathrm{SU}(N) / Z_{N}\right]=Z_{N}$. The model therefore possesses an $N$-fold vacuum degeneracy, and there is a discrete vacuum angle analogous to the $\theta$ parameter of QCD $[13,14]$. In addition, for $N=2$ there is expected to be a nonvanishing bilinear condensate [15].

The goal of the present work is to understand how this structure arises in the light-front framework. As we shall see, if proper attention is paid to the subtleties of light-front quantization, then the expected features can all be correctly reproduced. In particular, for $N=2$ we shall explicitly exhibit the $Z_{2}$ vacuum degeneracy and find a nonzero condensate. In the light-front representation the vacuum states can be described completely, unlike in the equal-time approach. 
However, the condensate we obtain is proportional to $1 / L$, where $L$ is the periodicity length, and so vanishes in the infinite-volume limit. This behavior is familiar from the Schwinger model and may be traced to the infrared regulator we employ. We shall discuss these issues further below.

Interestingly, for massless fermions the spectrum of massive states of the adjoint model has recently been shown to be identical to that of $(1+1)$-dimensional Yang-Mills theory with multiple flavors of fundamental fermions [16]. For this to be true it is necessary that the massive spectrum be independent of the vacuum angle that enters in the construction of the physical ground state. We will show this explicitly. In fact, the only quantity which depends on the vacuum angle is the chiral condensate, much like in the Schwinger model.

We shall begin by reviewing the essentials of the lightfront solution of the Schwinger model presented in Refs. [11]. This will serve to introduce the basic framework and to highlight the issues that are central to the occurrence of nontrivial vacuum structure in the light-front representation. We then discuss the formulation of SU(2) gauge theory with adjoint fermions. We shall consider a formulation of the theory in which the gauge field zero mode is treated as a constrained variable; a complementary formalism, in which the vacuum contains a dynamical zero mode content, is discussed in Ref. [17]. We show that this model possesses degenerate vacuum states which we calculate explicitly. The physical ground state is a superposition of these constructed to satisfy the cluster property. Next we compute the condensate, the expectation value of $\bar{\Psi} \Psi$ in this state, and briefly discuss the $L$ dependence of the result. Finally, we touch on some unresolved issues and directions for future work.

\section{THE SCHWINGER MODEL}

The Schwinger model is electrodynamics of massless fermions in $1+1$ dimensions [18]. The present discussion will necessarily be rather telegraphic, as our aim is mainly to highlight the issues that will be important later. For further details the reader is advised to consult [11].

To begin with, let us consider a free massless fermion. We shall employ the convention $x^{ \pm}=\left(x^{0} \pm x^{1}\right) / \sqrt{2}$, and decompose the Fermi field in the usual way:

$$
\psi_{ \pm} \equiv \frac{1}{\sqrt{2}} \gamma^{0} \gamma^{ \pm} \psi
$$

In $1+1$ dimensions (only) these are the same as chiral projections, so that

$$
\psi_{+}=\left(\begin{array}{c}
\psi_{R} \\
0
\end{array}\right), \quad \psi_{-}=\left(\begin{array}{c}
0 \\
\psi_{L}
\end{array}\right) .
$$

Now, the need to include degrees of freedom on two different lightlike lines can be seen immediately from the equation of motion, which takes the form

$$
\partial_{+} \psi_{R}=\partial_{-} \psi_{L}=0,
$$

where $\partial_{ \pm} \equiv \partial / \partial x^{ \pm}$. These have as their general solution

$$
\psi_{R}=f\left(x^{-}\right), \quad \psi_{L}=g\left(x^{+}\right),
$$

where $f$ and $g$ are arbitrary. Clearly, information along lines of both constant $x^{+}$and $x^{-}$must be specified to obtain a general solution to the Dirac equation. If in the quantum theory we do not include degrees of freedom to represent all of this freedom of the classical solution space, then the resulting theory will be incomplete.

The proper light-front formulation of this theory involves a pair of independent fields: $\psi_{R}$, initialized on $x^{+}=0$, and $\psi_{L}$, initialized on $x^{-}=0$. We regulate the infrared behavior by requiring that $\psi_{R / L}$ satisfy antiperiodic boundary conditions in $0 \leqslant x^{\mp} \leqslant 2 L$. $^{2}$ We can then Fourier expand the fields on their respective initial-value surfaces:

$$
\begin{aligned}
& \psi_{R}\left(0, x^{-}\right)=\frac{1}{2^{1 / 4} \sqrt{2 L}} \sum_{n=1 / 2}^{\infty}\left(b_{n} e^{-i k_{n}^{+} x^{-}}+d_{n}^{\dagger} e^{i k_{n}^{+} x^{-}}\right), \\
& \psi_{L}\left(x^{+}, 0\right)=\frac{1}{2^{1 / 4} \sqrt{2 L}} \sum_{n=1 / 2}^{\infty}\left(\beta_{n} e^{-i k_{n}^{-} x^{+}}+\delta_{n}^{\dagger} e^{i k_{n}^{-} x^{+}}\right),
\end{aligned}
$$

where $k_{n}^{ \pm}=n \pi / L$ and the sums are over odd half integers. Throughout this paper we shall use lower-case (upper-case) letters to denote indices that take odd half-integer (integer) values. The canonical anticommutation relations are

$$
\begin{aligned}
& \left\{\psi_{R}\left(0, x^{-}\right), \psi_{R}^{\dagger}\left(0, y^{-}\right)\right\}=\frac{1}{\sqrt{2}} \delta\left(x^{-}-y^{-}\right), \\
& \left\{\psi_{L}\left(x^{+}, 0\right), \psi_{L}^{\dagger}\left(y^{+}, 0\right)\right\}=\frac{1}{\sqrt{2}} \delta\left(x^{+}-y^{+}\right) .
\end{aligned}
$$

These are realized by the Fock algebra

$$
\left\{b_{n}, b_{m}^{\dagger}\right\}=\left\{d_{n}, d_{m}^{\dagger}\right\}=\left\{\beta_{n}, \beta_{m}^{\dagger}\right\}=\left\{\delta_{n}, \delta_{m}^{\dagger}\right\}=\delta_{m, n},
$$

with all other anticommutators vanishing. The Fock space is generated by applying the various creation operators to a vacuum state $|0\rangle$.

An important feature of this construction is that the dynamical operators $P^{ \pm}$, and in fact all conserved charges, receive contributions from both parts of the initial-value surface. This follows from very general considerations [20]. We have

$$
P^{ \pm}=\int_{0}^{2 L} d x^{-} \Theta^{+ \pm}+\int_{0}^{2 L} d x^{+} \Theta^{- \pm}
$$

where the second term accounts for the energy momentum of the left movers.

Let us now turn to the Schwinger model. The classical Lagrangian density is

\footnotetext{
${ }^{2}$ Note that in general the initial-value surface should be chosen so as to contain no points that are separated by timelike intervals; in such a case the commutation relations of the fields could not in general be known a priori. For a detailed discussion of these issues in the light-front context see [19].
} 


$$
\mathcal{L}=\frac{1}{2}\left[i \bar{\psi} \gamma^{\mu} \partial_{\mu} \psi-i\left(\partial_{\mu} \bar{\psi}\right) \gamma^{\mu} \psi\right]-\frac{1}{4} F^{\mu \nu} F_{\mu \nu}-e A^{\mu} J_{\mu}
$$

where $F_{\mu \nu}=\partial_{\mu} A_{\nu}-\partial_{\nu} A_{\mu}$ and $J^{\mu}=\bar{\psi} \gamma^{\mu} \psi$. We shall impose periodic boundary conditions in $x^{-}$on $A_{\mu}$, and choose the gauge $\partial_{-} A^{+}=0$. Note that the light-cone gauge $A^{+}=0$ is not allowed in the presence of the nontrivial spatial topology [21]. Thus $A^{+} \equiv v$, a zero mode. To simplify the notation let us further introduce $A \equiv A^{-}$. The equations of motion then take the form

$$
\begin{gathered}
-\partial_{-}^{2} A=e J^{+} \equiv e J^{R}, \\
\partial_{+} \partial_{-} A=e J^{-} \equiv e J^{L}, \\
\left(\partial_{+}+i e A\right) \psi_{R}=0, \\
\left(\partial_{-}+i e v\right) \psi_{L}=0,
\end{gathered}
$$

where $J^{R}=\sqrt{2} \psi_{R}^{\dagger} \psi_{R}$ and $J^{L}=\sqrt{2} \psi_{L}^{\dagger} \psi_{L}$.

We should perhaps elaborate somewhat on the choice of gauge. The gauge field is chosen to be periodic in $x^{-}$but satisfies no particular boundary condition in $x^{+}$. Thus the gauge transformation required to bring an arbitrary configuration $A_{\mu}\left(x^{+}, x^{-}\right)$to one satisfying $\partial_{-} A^{+}=0$ will in general not be periodic in $x^{+}$, and so will violate the boundary condition we have imposed on $\psi_{L}$. However, after such a transformation we can apply a purely $x^{+}$-dependent gauge transformation that restores the antiperiodicity of $\psi_{L}$ at a single value of $x^{-}$, which we can choose to be the initial-value surface $x^{-}=0$. This does not affect the boundary conditions satisfied by the other fields. To be precise, therefore, we should say that we require that $\psi_{L}$ be antiperiodic on its initial-value surface only; it may not remain antiperiodic as it evolves in $x^{-}$. (Exactly what happens to $\psi_{L}$ is discovered by solving its equation of motion.) This condition, and the conditions imposed on $\psi_{R}$ and $A_{\mu}$, are then consistent with the gauge choice $\partial_{-} A^{+}=0$.

Next let us discuss the definition of singular operator products, as this is central to the issue of vacuum structure. We define the current operators by a gauge-invariant point splitting:

$$
\begin{aligned}
J^{R}\left(0, x^{-}\right) \equiv & \sqrt{2} \lim _{\epsilon^{-} \rightarrow 0}\left[\exp \left(-i e \int_{x}^{x+\epsilon^{-}} v d x^{-}\right)\right. \\
& \left.\times \psi_{R}^{\dagger}\left(0, x^{-}+\epsilon^{-}\right) \psi_{R}\left(0, x^{-}\right)-\mathrm{VEV}\right], \\
J^{L}\left(x^{+}, 0\right) \equiv & \sqrt{2} \lim _{\epsilon^{+} \rightarrow 0}\left[\exp \left(-i e \int_{x}^{x+\epsilon^{+}} A d x^{+}\right)\right. \\
& \left.\times \psi_{L}^{\dagger}\left(x^{+}+\epsilon^{+}, 0\right) \psi_{L}\left(x^{+}, 0\right)-\mathrm{VEV}\right] .
\end{aligned}
$$

Note that we must split $\psi_{R}^{\dagger} \psi_{R}$ in the $x^{-}$direction and $\psi_{L}^{\dagger} \psi_{L}$ in the $x^{+}$direction. This follows from the canonical singularity structure of the fields [Eqs. (2.7) and (2.8)]. Evaluating the singularities in the operator products as $\epsilon^{ \pm} \rightarrow 0$ we find

$$
\begin{aligned}
& J^{R}=\widetilde{J}^{R}-\frac{e}{2 \pi} v, \\
& J^{L}=\widetilde{J}^{L}-\frac{e}{2 \pi} A,
\end{aligned}
$$

where $\widetilde{J}^{ \pm}$are the "naive" normal-ordered currents. It will be useful to express these in terms of their Fourier modes (the so-called "fusion operators"). We write

$$
\begin{aligned}
& \widetilde{J}^{R}=\frac{1}{2 L_{N}} \sum_{=-\infty}^{\infty} C_{N} e^{-i k_{N}^{+} x^{-}}, \\
& \widetilde{J}^{L}=\frac{1}{2 L_{N}} \sum_{N=-\infty}^{\infty} D_{N} e^{-i k_{N}^{-} x^{+}},
\end{aligned}
$$

where the sums run over the integers. For $N=0$ these are the charge operators for the right and left movers,

$$
\begin{aligned}
& C_{0}=\sum_{n=1 / 2}^{\infty}\left(b_{n}^{\dagger} b_{n}-d_{n}^{\dagger} d_{n}\right), \\
& D_{0}=\sum_{n=1 / 2}^{\infty}\left(\beta_{n}^{\dagger} \beta_{n}-\delta_{n}^{\dagger} \delta_{n}\right),
\end{aligned}
$$

while for $N>0$ they are given by

$$
\begin{aligned}
& C_{N}=\sum_{n=1 / 2}^{\infty}\left(b_{n}^{\dagger} b_{N+n}-d_{n}^{\dagger} d_{N+n}\right)+\sum_{n=1 / 2}^{N-1 / 2} d_{N-n} b_{n}, \\
& D_{N}=\sum_{n=1 / 2}^{\infty}\left(\beta_{n}^{\dagger} \beta_{N+n}-\delta_{n}^{\dagger} \delta_{N+n}\right)+\sum_{n=1 / 2}^{N-1 / 2} \delta_{N-n} \beta_{n} .
\end{aligned}
$$

For $N<0$ they may be obtained by conjugation:

$$
C_{-N}=C_{N}^{\dagger}, \quad D_{-N}=D_{N}^{\dagger} .
$$

They can be shown to satisfy the simple algebra

$$
\left[C_{M}, C_{N}\right]=\left[D_{M}, D_{N}\right]=M \delta_{M,-N} .
$$

We can now discuss the implementation of Gauss' law, Eq. (2.12). Projected onto the normal-mode sector (in $x^{-}$), this is a constraint which determines the normal modes of $A$ on $x^{+}=0$ in the usual way. Projected onto the zero-mode sector we obtain

$$
0=C_{0}-z_{R},
$$

where $z_{R} \equiv e v L / \pi$. This can be interpreted in at least two ways. One obvious possibility is to just use Eq. (2.28) to determine $z_{R}$ in terms of the charge operator (although there is a subtlety that arises which we shall explain in a moment). If we wish to treat $z_{R}$ as a dynamical field, however, then Eq. (2.28) cannot hold as an operator relation; it must be imple- 
mented via restriction to some physical subspace. The principal difficulty with this approach arises from the fact that $z_{R}$ is $x^{+}$dependent while $C_{0}$ is not. Thus the operator we would like to use to select physical states does not commute with the Hamiltonian $P^{-}$, and the stability of the physical subspace is unclear. It is immediately obvious, for example, that the vanishing of the right-hand side of Eq. (2.28) cannot be imposed as an eigenvalue condition, if there are to be eigenstates of the Hamiltonian in the physical subspace. It may be possible to realize this condition in matrix elements between suitably defined physical states, however.

Here we shall interpret Eq. (2.28) as determining $z_{R}$ in terms of the charge. There is a subtlety in solving Eq. (2.28) as it stands, however. We shall see below that $z_{R}$ should not be taken to be a function of $\psi_{R}$, as this would lead to inconsistencies in the Heisenberg equations. Anticipating that the physical subspace of the Schwinger model will consist of states with vanishing total charge $C_{0}+D_{0}$, let us rewrite Eq. (2.28) in the form

$$
0=C_{0}+D_{0}-D_{0}-z_{R} .
$$

We can then take

$$
z_{R}=-D_{0},
$$

which gives no conflict with the Heisenberg equations, and what remains is the expected condition defining physical states. Thus the zero mode of Gauss' law is interpreted as determining $z_{R}$ in terms of $D_{0}$, and giving the condition $C_{0}+D_{0}=0$, which must be imposed as an eigenvalue condition on the states. This construction may seem somewhat $a d$ hoc but in fact it can be justified by careful consideration of the proper coupling of the gauge field to matter [11]. The situation is really the same as in classical electromagnetism.

The zero mode of Ampere's law, Eq. (2.13), may be analyzed similarly, leading to

$$
z_{L}=-C_{0},
$$

where $z_{L} \equiv e w L / \pi$ and $w$ is the zero mode of $A$, and the same neutrality condition on the states, $C_{0}+D_{0}=0$.

Next let us construct the Poincaré generators $P^{ \pm}$. As discussed above, these are given as integrals of the appropriate components of the energy-momentum tensor over both pieces of the initial-value surface. If we work out $\Theta^{\mu \nu}$ as usual and try to evaluate Eq. (2.10), however, we encounter a difficulty: the integral over $x^{+}$involves fields that are initialized on the surface $x^{+}=0$. But we do not know these fields as functions of $x^{+}$until we have solved the theory. There is a simple way of dealing with this which works, at least for the Schwinger model. We work out $\Theta^{\mu \nu}$ as usual, but include in the calculation of $P^{ \pm}$only those terms that contain quantities we know on the different parts of the initial-value surface.

No proof exists of the correctness of this procedure, but any alleged results for $P^{ \pm}$can be checked a posteriori for correctness, by verifying that they properly translate all the fields in $x^{ \pm}$and satisfy the Poincare algebra. The same is true of other operators, for example, the charges or the boost generator-we can check in the end whether the expressions we take are the correct ones. We will approach the problem of constructing $P^{ \pm}$from this practical perspective: we start from the canonical energy-momentum tensor, but modify it as necessary in response to any problems of consistency which arise. In the end we shall justify the final expressions by showing that they satisfy all necessary criteria.

Let us first consider $P^{+}$. The relevant components of the energy-momentum tensor, derived via the standard Noether procedure, are

$$
\begin{gathered}
\Theta^{++}=i \sqrt{2} \psi_{R}^{\dagger} \partial_{-} \psi_{R} \\
\Theta^{-+}=-i \sqrt{2} \psi_{R}^{\dagger} \partial_{+} \psi_{R}+e\left(J^{R} A+J^{L} v\right)
\end{gathered}
$$

Using the rule stated above the only contribution comes from $\Theta^{++}$, so that

$$
P^{+}=i \sqrt{2} \int_{0}^{2 L} d x^{-} \psi_{R}^{\dagger} \partial_{-} \psi_{R}
$$

The operator product that occurs is singular, however, and must be regulated. We shall again split the product in $x^{-}$, introducing an eikonal factor to maintain gauge invariance:

$$
\begin{aligned}
\psi_{R}^{\dagger} \partial_{-} \psi_{R} \equiv & \lim _{\epsilon^{-} \rightarrow 0}\left[\exp \left(-i e \int_{x^{-}}^{x^{-}+\epsilon^{-}} d y^{-} v\right)\right. \\
& \left.\times \psi_{R}^{\dagger}\left(x^{-}+\epsilon^{-}\right) \partial_{-} \psi_{R}\left(x^{-}\right)-\mathrm{VEV}\right] .
\end{aligned}
$$

(Note that in some cases a symmetric splitting may be necessary to maintain the hermiticity of the regulated operator.) Evaluating the singularity in $\psi_{R}^{\dagger} \partial_{-} \psi_{R}$ as $\epsilon^{-} \rightarrow 0$ we then obtain

$$
P^{+}=P_{\text {free }}^{+}-\frac{\pi}{2 L} z_{R}^{2}
$$

where $P_{\text {free }}^{+}$is the free-particle momentum operator for the right movers:

$$
P_{\text {free }}^{+}=\sum_{n}\left(\frac{n \pi}{L}\right)\left[b_{n}^{\dagger} b_{n}+d_{n}^{\dagger} d_{n}\right] \text {. }
$$

It is now possible to see why it would be dangerous to have $z_{R}$ be a function of the right movers, through $C_{0}$, for example. The operator $P^{+}$should generate translations of $\psi_{R}$ in its initial-value surface via

$$
\left[\psi_{R}, P^{+}\right]=i \partial_{-} \psi_{R} .
$$

But this is already accomplished by $P_{\text {free }}^{+}$, and any additional terms in $P^{+}$should commute with $\psi_{R}$ to avoid spoiling this relation. This is what leads us to the modification of the zero-mode part of Gauss' law discussed above, and to the solution (2.30) for $z_{R}$ in terms of $D_{0}$.

In fact, Eq. (2.30) is essentially forced on us by considering the Heisenberg equation corresponding to Eq. (2.15). We must have

$$
\left[\psi_{L}, P^{+}\right]=\frac{1}{2 L}\left(z_{R} \psi_{L}+\psi_{L} z_{R}\right),
$$


or equivalently

$$
\left[\psi_{L}, z_{R}\right]=-\psi_{L}
$$

This requirement, along with $\left[\psi_{R}, z_{R}\right]=0$, essentially fixes $z_{R}$ to be given by Eq. (2.30). Thus $P^{+}$takes the final form

$$
P^{+}=P_{\text {free }}^{+}-\frac{\pi}{2 L} D_{0}^{2}
$$

Note the minus sign in front of the second term. This is crucial for the appearance of nontrivial vacuum structure, as it allows certain states containing left movers to be degenerate with the Fock vacuum $|0\rangle$.

If we treat $z_{R}$ as dynamical, then Eq. (2.36) is actually inconsistent as it stands. This is because $\pi_{R}$, the momentum conjugate to $z_{R}$, does not commute with this $P^{+}$. But it must commute with $P^{+}$, since it is itself a zero mode, i.e., independent of $x^{-}$, and $P^{+}$generates translations on $x^{-}$. This problem may be solved by modifying $P^{+}$, but it is interesting to note that a naive application of point splitting appears to be inconsistent with treating $z_{R}$ as dynamical.

The Hamiltonian $P^{-}$can be constructed similarly. The only subtlety here involves the gauge correction of the singular operator product occurring in

$$
i \sqrt{2} \int_{0}^{2 L} d x^{+} \psi_{L}^{\dagger} \partial_{+} \psi_{L}
$$

Because this product is split in the $x^{+}$direction, the eikonal factor involves $A$. But most of $A$ is unknown on the surface $x^{-}=0$; its normal modes are constrained functionals of $\psi_{R}$, determined by solving Gauss' law. The solution to this difficulty is to keep in the expression for $P^{-}$only that part of $A$ that we do know on $x^{-}=0$, namely its zero mode (2.31). (That $C_{0}$ is $x^{+}$independent will be checked momentarily.) With this ansatz we arrive at

$$
P^{-}=P_{\text {free }}^{-}-\frac{\pi}{2 L} C_{0}^{2}+\frac{e^{2} L}{2 \pi^{2}} \sum_{N=-\infty}^{\infty}{ }^{\prime}\left(\frac{1}{N^{2}}\right) C_{N} C_{-N},
$$

where the prime indicates that the term with $N=0$ is omitted and $P_{\text {free }}^{-}$is the free energy of the left movers:

$$
P_{\text {free }}^{-}=\sum_{n}\left(\frac{n \pi}{L}\right)\left[\beta_{n}^{\dagger} \beta_{n}+\delta_{n}^{\dagger} \delta_{n}\right]
$$

It is clear that this $P^{-}$commutes with $C_{0}$, so that $\partial_{+} C_{0}=0$ as promised.

At this stage we have dynamical operators that correctly translate all the fermionic degrees of freedom in $x^{ \pm}$, and are consistent with the solutions we took for the zero modes of the gauge field. Gauss' law is also satisfied by construction. The only issue that remains is whether or not Ampere's law, Eq. (2.13), is satisfied.

This is straightforward to check. The zero mode of Ampere's law reduces to the condition $C_{0}+D_{0}=0$, which defines the physical subspace. To check the normal mode part, we take the operator $\partial_{-} A$ obtained from solving Gauss' law and commute it with $P^{-}$to obtain its $x^{+}$derivative. It is straightforward to check that this results in an equality, except that the terms in $J^{L}$ involving the $D_{N}$ are not reproduced in the commutator. Thus Ampere's law is only obtained in matrix elements between states that satisfy

$$
D_{N}|\Phi\rangle=0 \quad(N>0)
$$

This must be added to the conditions defining physical states.

This is a very important result, as it removes most of the states with left-moving quanta from the physical subspace. In fact, it can be shown [11] that the only states with left movers that remain in the physical subspace are the states

$$
\begin{aligned}
& \left|V_{N}\right\rangle=d_{N-1 / 2}^{\dagger} \beta_{N-1 / 2}^{\dagger} \cdots d_{1 / 2}^{\dagger} \beta_{1 / 2}^{\dagger}|0\rangle, \\
& \left|V_{-N}\right\rangle=b_{N-1 / 2}^{\dagger} \delta_{N-1 / 2}^{\dagger} \cdots b_{1 / 2}^{\dagger} \delta_{1 / 2}^{\dagger}|0\rangle,
\end{aligned}
$$

where $N=1,2, \ldots$, and states created by applying functionals of $\psi_{R}$ to these. It is straightforward to check that these are all eigenstates of $P^{ \pm}$with eigenvalue zero, so that they are degenerate with the bare vacuum. The physical ground state of the theory is a superposition of these, which can be shown to be necessary to satisfy the requirement of cluster decomposition:

$$
|\theta\rangle=\sum_{N=-\infty}^{\infty} e^{-i N \theta}\left|V_{N}\right\rangle
$$

Thus we recover the expected $\theta$ structure of the physical vacuum state in this model.

The full solution of the theory is straightforward given the form of $P^{-}$. We first verify that the states obtained by acting with the $C_{N}$ on $|\theta\rangle$ span the physical subspace. We then define creation and destruction operators from the positive and negative frequency $C_{N}$, respectively. Equation (2.27) is then a bosonic canonical commutator and the Hamiltonian is diagonal. In the end we recover the well-known results that the physical states correspond to noninteracting bosons of mass $e / \sqrt{\pi}$. In addition, there is a nonvanishing condensate $\langle\theta|\bar{\psi} \psi| \theta\rangle$ with the correct dependence on $\theta$. One can also check that the correct chiral anomaly is obtained. These and other issues are discussed in detail in [11].

Our goal in this section was to use the Schwinger model to highlight the issues that will be important when we discuss the non-Abelian theory. The main lessons to be drawn concern the need to include degrees of freedom initialized on two different null planes, and the regulation of singular operator products in a gauge-invariant way. In addition, we must construct dynamical operators that are consistent with the equations of motion and initial conditions, and identify a subspace in which those equations of motion that are not satisfied as operator relations can hold. Finally, we must verify that this physical subspace is stable under evolution in $x^{ \pm}$.

\section{SU(2) GAUGE THEORY COUPLED TO ADJOINT FERMIONS: BASICS}

Let us now consider $\mathrm{SU}(2)$ gauge theory coupled to massless adjoint fermions in $1+1$ dimensions. The Lagrangian density for the theory is 


$$
\mathcal{L}=-\frac{1}{2} \operatorname{Tr}\left(F^{\mu \nu} F_{\mu \nu}\right)+\frac{i}{2} \operatorname{Tr}\left(\overline{\psi \gamma}{ }^{\mu} \stackrel{\leftrightarrow}{D}_{\mu} \psi\right),
$$

where $\quad D_{\mu}=\partial_{\mu}+i g\left[A_{\mu},\right] \quad$ and $\quad F_{\mu \nu}=\partial_{\mu} A_{\nu}-\partial_{\nu} A_{\mu}$ $+i g\left[A_{\mu}, A_{\nu}\right]$. A convenient representation for the $\gamma$ matrices is $\gamma^{0}=\sigma^{2}$ and $\gamma^{1}=i \sigma^{1}$, where $\sigma^{a}$ are the Pauli matrices. With this choice the (Majorana) Fermi field may be taken to be Hermitian.

The matrix representation of the fields makes use of the fundamental $\mathrm{SU}(2)$ generators $\tau^{a}=\sigma^{a} / 2$. It is convenient to introduce a color helicity, or Cartan, basis, defined by

$$
\tau^{ \pm} \equiv \frac{1}{\sqrt{2}}\left(\tau^{1} \pm i \tau^{2}\right)
$$

with $\tau^{3}$ unchanged. These satisfy

$$
\begin{gathered}
{\left[\tau^{+}, \tau^{-}\right]=\tau^{3},} \\
{\left[\tau^{3}, \tau^{ \pm}\right]= \pm \tau^{ \pm} .}
\end{gathered}
$$

Lower helicity indices are defined by $\tau_{ \pm}=\tau^{\mp}$, and matrixvalued fields are decomposed as, for example,

$$
A^{\mu}=A_{3}^{\mu} \tau^{3}+A_{+}^{\mu} \tau^{+}+A_{-}^{\mu} \tau^{-},
$$

where $A^{\mu, \pm} \equiv\left(A_{1}^{\mu} \pm i A_{2}^{\mu}\right) / \sqrt{2}$ and $A^{\mu, \pm}=A_{+}^{\mu}$. [Note also that $\left(A_{+}^{\mu}\right)^{\dagger}=A_{-}^{\mu}$.] The Fermi field will be written as

$$
\Psi_{R / L}=\psi_{R / L} \tau^{3}+\phi_{R / L} \tau^{+}+\phi_{R / L}^{\dagger} \tau^{-},
$$

where $\phi_{R / L} \equiv\left(\Psi_{R / L}^{1}-i \Psi_{R / L}^{2}\right) / \sqrt{2}$ and the labels $R / L$ indicate light-front spinor projections as given in Eq. (2.2). Note that under a gauge transformation the Fermi field transforms according to

$$
\Psi_{R / L}^{\prime}=U \Psi_{R / L} U^{-1},
$$

where $U$ is a spacetime-dependent element of $\mathrm{SU}(2)$.

We shall regulate the theory in the infrared by imposing certain boundary conditions in $x^{ \pm}$. The fields $\phi_{R}$ and $\phi_{L}$ will be taken to be antiperiodic in $x^{-}$and $x^{+}$, respectively. It will be convenient, however, to take $\psi_{R}$ and $\psi_{L}$ to be periodic in $x^{-}$and $x^{+}$, respectively ("twisted" boundary conditions). The reasons for this will become clear as we progress. For consistency, then, $A_{ \pm}^{\mu}$ must be taken to be antiperiodic in $x^{-}$, while $A_{3}^{\mu}$ is periodic. In all cases the periodicity length is $2 L$.

The Fock representation for the fermionic degrees of freedom is obtained by Fourier expanding $\Psi_{R}$ on $x^{+}=0$ and $\Psi_{L}$ on $x^{-}=0$. We have

$$
\begin{gathered}
\psi_{R}\left(0, x^{-}\right)=\frac{1}{2^{1 / 4} \sqrt{2 L}} \sum_{N=1}^{\infty}\left(a_{N} e^{-i k_{N}^{+} x^{-}}+a_{N}^{\dagger} e^{i k_{N}^{+} x^{-}}\right)+\stackrel{\circ}{\psi}_{R}, \\
\phi_{R}\left(0, x^{-}\right)=\frac{1}{2^{1 / 4} \sqrt{2 L}} \sum_{n=1 / 2}^{\infty}\left(b_{n} e^{-i k_{n}^{+} x^{-}}+d_{n}^{\dagger} e^{i k_{n}^{+} x^{-}}\right),
\end{gathered}
$$

$$
\psi_{L}\left(x^{+}, 0\right)=\frac{1}{2^{1 / 4} \sqrt{2 L}} \sum_{N=1}^{\infty}\left(\alpha_{N} e^{-i k_{N}^{-} x^{+}}+\alpha_{N}^{\dagger} e^{i k_{N}^{-} x^{+}}\right)+\stackrel{\circ}{\psi}_{L},
$$

$$
\phi_{L}\left(x^{+}, 0\right)=\frac{1}{2^{1 / 4} \sqrt{2 L}} \sum_{n=1 / 2}^{\infty}\left(\beta_{n} e^{-i k_{n}^{-} x^{+}}+\delta_{n}^{\dagger} e^{i k_{n}^{-} x^{+}}\right),
$$

where we have explicitly separated out the zero modes of $\psi_{R / L}$. As before, the lower-case (upper-case) indices run over positive half-odd integers (integers) and $k_{n}^{ \pm}=n \pi / L$. The Fourier modes obey the algebra

$$
\begin{gathered}
\left\{a_{N}^{\dagger}, a_{M}\right\}=\left\{\alpha_{N}^{\dagger}, \alpha_{M}\right\}=\delta_{N, M}, \\
\left\{b_{n}^{\dagger}, b_{m}\right\}=\left\{d_{n}^{\dagger}, d_{m}\right\}=\left\{\beta_{n}^{\dagger}, \beta_{m}\right\}=\left\{\delta_{n}^{\dagger}, \delta_{m}\right\}=\delta_{n, m}, \\
\left\{\stackrel{\circ}{\psi}_{R}, \stackrel{\circ}{\psi}_{R}\right\}=\left\{\stackrel{\circ}{\psi}_{L}, \stackrel{\circ}{\psi}_{L}\right\}=\frac{1}{2 \sqrt{2} L},
\end{gathered}
$$

with all mixed anticommutators vanishing. These are equivalent to the canonical anti-commutation relations

$$
\begin{aligned}
& \left\{\Psi_{R}\left(0, x^{-}\right), \Psi_{R}\left(0, y^{-}\right)\right\}=\frac{1}{\sqrt{2}} \delta\left(x^{-}-y^{-}\right), \\
& \left\{\Psi_{L}\left(x^{+}, 0\right), \Psi_{L}\left(y^{+}, 0\right)\right\}=\frac{1}{\sqrt{2}} \delta\left(x^{+}-y^{+}\right) .
\end{aligned}
$$

The fermionic Fock space is generated by acting with the various creation operators on a vacuum state $|0\rangle$.

For simplicity, in the remainder of this paper we shall discard the zero modes of $\psi_{R / L}$. It can be shown that including them does not qualitatively affect any of our results; they merely complicate parts of the analysis. In addition, their physical meaning is somewhat ambiguous. For example, they lead to a nonvanishing fermion condensate even in free field theory. This same phenomenon was observed in the equal-time context in Ref. [22]. We shall therefore simply exclude them from the model; the condensate we obtain is then entirely an effect of the interaction. Note that this truncation does not lead to inconsistencies in the model. For example, the Heisenberg equations for $\psi_{R / L}$ will simply reduce to the appropriate Euler-Lagrange equations with the zero modes removed. In addition, the Poincare algebra is unaffected.

The current operators for this theory are

$$
\begin{aligned}
& J^{+} \equiv J^{R}=-\frac{1}{\sqrt{2}}\left[\Psi_{R}, \Psi_{R}\right], \\
& J^{-} \equiv J^{L}=-\frac{1}{\sqrt{2}}\left[\Psi_{L}, \Psi_{L}\right] .
\end{aligned}
$$

To avoid confusion, we shall henceforth always write the currents with $R$ or $L$ in place of the upper Lorentz index. These expressions are ill defined as they stand since they 
contain products of operators at the same point. This is a common problem and occurs in the expressions for the Poincaré generators as well. We shall regulate these by point splitting, introducing an eikonal factor to maintain gauge invariance, and then taking the splitting to zero after removing the divergences. One can show, for example, that

$$
\begin{aligned}
& {\left[\exp \left(i g \int_{x}^{x+\epsilon^{\mu}} A \cdot d x\right) \Psi\left(x+\epsilon^{\mu}\right)\right.} \\
& \left.\quad \times \exp \left(-i g \int_{x}^{x+\epsilon^{\mu}} A \cdot d x\right), \Psi(x)\right]
\end{aligned}
$$

transforms covariantly in the adjoint representation. In the limit of small $\epsilon^{\mu}$ one effectively replaces

$$
\Psi(x) \rightarrow \Psi\left(x+\epsilon^{\mu}\right)+i g\left[A \cdot \epsilon, \Psi\left(x+\epsilon^{\mu}\right)\right] .
$$

The singularity in the Fermi operator product as $\epsilon \rightarrow 0$ picks up the $\epsilon$ in the above expression leaving an additional contribution. The splitting must be performed in the $x^{-}$direction for $\Psi_{R}$ and in the $x^{+}$direction for $\Psi_{L}$. A straightforward calculation gives

$$
\begin{aligned}
& J^{R}=\widetilde{J}^{R}-\frac{g}{2 \pi} A^{+}, \\
& J^{L}=\widetilde{J}^{L}-\frac{g}{2 \pi} A^{-},
\end{aligned}
$$

where $\widetilde{J}^{R / L}$ are the naive normal ordered currents.

In the helicity basis the components of $\widetilde{J}^{R}$ take the forms

$$
\begin{aligned}
& \widetilde{J}_{3}^{R}=: \frac{1}{\sqrt{2}}\left(\phi_{R}^{\dagger} \phi_{R}-\phi_{R} \phi_{R}^{\dagger}\right):, \\
& \widetilde{J}_{-}^{R}=: \frac{1}{\sqrt{2}}\left(\psi_{R} \phi_{R}^{\dagger}-\phi_{R}^{\dagger} \psi_{R}\right):, \\
& \widetilde{J}_{+}^{R}=: \frac{1}{\sqrt{2}}\left(\phi_{R} \psi_{R}-\psi_{R} \phi_{R}\right):
\end{aligned}
$$

The corresponding expressions for the components of $\widetilde{J}^{L}$ are identical, with $R \rightarrow L$. It is convenient to Fourier expand these currents and discuss the properties of their components. We write

$$
\begin{aligned}
& \widetilde{J}^{R, a}=\frac{1}{2 L} \sum_{N=-\infty}^{\infty} C_{N}^{a} e^{-i \pi N x^{-} / L}, \\
& \widetilde{J}^{L, a}=\frac{1}{2 L_{N}} \sum_{N=-\infty}^{\infty} D_{N}^{a} e^{-i \pi N x^{+} / L},
\end{aligned}
$$

where $a$ is a color index and the sums run over integers for $a=3$ and half-odd integers for $a= \pm$. It is well known that the Fourier components satisfy a Kac-Moody algebra with level two [23]. We shall discuss this explicitly for the $C_{N}^{a}$; with appropriate substitutions an identical set of relations holds for the $D_{N}^{a}$.
In terms of the Fock operators we have, for $N, n>0$,

$$
\begin{gathered}
C_{N}^{3}=\sum_{n=1 / 2}^{\infty}\left(b_{n}^{\dagger} b_{N+n}-d_{n}^{\dagger} d_{N+n}\right)-\sum_{n=1 / 2}^{N-1 / 2} b_{n} d_{N-n}, \\
C_{n}^{+}=\sum_{M=1}^{\infty} a_{M}^{\dagger} d_{n+M}-\sum_{m=1 / 2}^{\infty} b_{m}^{\dagger} a_{n+m}-\sum_{m=1 / 2}^{n-1} d_{m} a_{n-m}, \\
C_{n}^{-}=\sum_{m=1 / 2}^{\infty} d_{m}^{\dagger} a_{n+m}-\sum_{M=1}^{\infty} a_{M}^{\dagger} b_{M+n}-\sum_{m=1 / 2}^{n-1} a_{n-m} b_{m} .
\end{gathered}
$$

We may obtain the modes for $N, n<0$ from the above by Hermitian conjugation:

$$
C_{-N}^{3}=\left(C_{N}^{3}\right)^{\dagger}, \quad C_{-n}^{+}=\left(C_{n}^{-}\right)^{\dagger}, \quad C_{-n}^{-}=\left(C_{n}^{+}\right)^{\dagger} .
$$

Finally, $C_{0}^{3}$ is just the three-color charge in the right-moving fermions:

$$
C_{0}^{3}=\sum_{n}\left(b_{n}^{\dagger} b_{n}-d_{n}^{\dagger} d_{n}\right) .
$$

In the Cartan basis, the Kac-Moody algebra takes the form

$$
\begin{gathered}
{\left[C_{N}^{3}, C_{M}^{3}\right]=N \delta_{N,-M},} \\
{\left[C_{n}^{ \pm}, C_{m}^{ \pm}\right]=0,} \\
{\left[C_{N}^{3}, C_{m}^{ \pm}\right]= \pm C_{N+m}^{ \pm},} \\
{\left[C_{n}^{+}, C_{m}^{-}\right]=C_{n+m}^{3}+n \delta_{n,-m} .}
\end{gathered}
$$

It is straightforward to verify these relations using the fundamental anticommutators (3.12) and (3.13). The algebra satisfied by the $D$ 's is of course identical.

\section{ZERO MODES AND GAUGE FIXING}

The main subtlety arising from the use of discretization as a regulator is in fixing the gauge. It is most convenient in light-front field theory to choose the light-cone gauge $A^{+}=0$. However, this is not possible with the boundary conditions we have imposed; since gauge transformations must be periodic up to an element of the center of the gauge group (here $Z_{2}$ ), we cannot gauge the zero mode of $A^{+}$to zero [21]. It is permissible to take $\partial_{-} A^{+}=0$, as in the Schwinger model. Having made that choice, there are two issues which arise involving the zero modes of the gauge field.

The first issue is whether the zero modes should be treated as independent degrees of freedom or as constrained functionals of the Fermi fields. In the equal-time representation this can usually be resolved on the basis of whether or not the Lagrangian gives a conjugate momentum for the operator in question. In the light-front representation the issue is more complex; in the Schwinger model, for example, the zero modes of the gauge fields are constrained even though the Lagrangian provides a conjugate momentum. 
Some of the difficulties in treating the zero mode $A^{+}$as a degree of freedom were mentioned in Sec. II. They arise principally because of Eq. (2.28), which cannot hold as an operator relation. Imposing it as a condition on states is somewhat delicate, however, since the stability of the physical subspace is not obvious. These and other aspects of the formulation with a dynamical zero mode are discussed in Ref. [17]. Here we shall avoid this difficulty by considering a formulation in which the zero mode is constrained.

In addition, $A^{+}$enters the operator $P^{+}$if we compute the Poincare generators according to the usual prescription. But if $A^{+}$is dynamical, then the resulting $P^{+}$does not commute with the momentum conjugate to $A^{+}$. This difficulty can perhaps be resolved by replacing the troublesome terms in $P^{+}$with operators to which they are weakly equivalent (i.e., equal in the physical subspace). Note that we must apply this procedure in the constrained case as well. If in the Schwinger model we had taken $z_{R}$ to be a functional of $\psi_{R}$ (possibly along with other things) we would have found an immediate contradiction between our definitions and the Heisenberg equations. Such a solution would be inconsistent even prior to any dynamical considerations. That is why in the Schwinger model $z_{R}$ is taken to be a functional of $\psi_{L}$ (although there are other ways to reach the same conclusion). The non-Abelian case turns out to be even more complicated, as we shall see.

The second issue involving the zero modes is the question of how to accomplish the residual gauge fixing using constant (in $x^{+}$) color matrices. In the equal-time representation, it is convenient to rotate the zero modes of $A^{+}$so that only the color 3 component is nonzero. Here we shall mostly be interested in the case where the zero modes of the gauge field are treated as constrained. In this case, since we should fix the gauge in terms of the degrees of freedom and let the constraint equations determine the constrained variables, we should proceed in a different way. The natural thing would presumably be to use the residual gauge freedom to rotate two components of the vector current to zero; probably that is the choice most nearly equivalent to rotating the gauge field zero modes in the equal-time representation. The problem is that this approach is technically difficult to implement. The Dirac-Bergmann procedure leads to a complicated nonlinear relation between the Fermi modes, due to the fact that the current is bilocal in the Fermi fields. A procedure which is very similar to the suggested gauge fixing, but is much simpler to carry out, is to use twisted boundary conditions for the Fermi fields [24,25] as we do here. Presumably the results of the Dirac-Bergmann procedure would be much the same if we were able to carry it out, but we have no proof of this. With these boundary conditions, the color 1 and 2 components of the gauge field must also be antiperiodic in $x^{-}$ and simply have no zero modes. Thus whether or not we treat the zero modes of $A^{+}$as constrained, we have

$$
A^{+}=v\left(x^{+}\right) \tau^{3}
$$

with $v$ independent of $x^{-}$. As we shall see, in the constrained case $v$ turns out to be independent of $x^{+}$as well.

While one could simultaneously rotate $A^{-} \equiv A$ so that it has no color 3 zero mode $[10,26]$, we shall not do that here.
Instead we will retain this zero mode, which we call $w$, and determine it in the solution of the equations of motion.

It is useful to introduce a set of transformations which are formally the "large", gauge transformations which connect different Gribov regions [27]. We shall denote these by $T_{N}^{R}$ and $T_{N}^{L}$, with $N$ any integer:

$$
\begin{gathered}
T_{N}^{R}=\exp \left[-\frac{i N \pi}{2 L} x^{-} \tau_{3}\right], \\
T_{N}^{L}=\exp \left[\frac{i N \pi}{2 L} x^{+} \tau_{3}\right] .
\end{gathered}
$$

It is convenient to define the dimensionless variables $z_{R}=g v L / \pi$ and $z_{L}=g w L / \pi$, which $T_{N}^{R / L}$ shifts by $\pm N$ :

$$
\begin{aligned}
& T_{N}^{R} z_{R}\left(T_{N}^{R}\right)^{-1}=z_{R}+N, \\
& T_{N}^{L} z_{L}\left(T_{N}^{L}\right)^{-1}=z_{L}-N .
\end{aligned}
$$

In addition, $T_{N}^{R / L}$ generates a spacetime-dependent phase rotation on the matter field $\phi_{R / L}$,

$$
\begin{gathered}
T_{N}^{R} \phi_{R}\left(T_{N}^{R}\right)^{-1}=e^{-i N \pi x^{-} / L} \phi_{R}, \\
T_{N}^{L} \phi_{L}\left(T_{N}^{L}\right)^{-1}=e^{i N \pi x^{+} / L} \phi_{L},
\end{gathered}
$$

which however preserves the boundary conditions on $\phi_{R / L}$. Note that for $T_{N}^{R / L}$ to be a symmetry of the theory, the solutions for $z_{R / L}$ in terms of the Fermi fields must correctly reproduce Eqs. (4.4) and (4.5) under the transformations (4.6) and (4.7). This will turn out to be the case.

The theory is also invariant under the so-called Weyl transformation, denoted $R$. This is also formally a gauge transformation, and takes

$$
\begin{gathered}
R z_{R / L} R^{-1}=-z_{R / L}, \\
R \phi_{R / L} R^{-1}=\phi_{R / L}^{\dagger}
\end{gathered}
$$

The action of $T_{1}^{R / L}$ and $R$ on the fermion Fock operators can be determined easily from Eqs. (4.6), (4.7), and (4.9). $T_{1}^{R}$ gives rise to a spectral flow for the right-handed particles,

$$
\begin{gathered}
T_{1}^{R} b_{n}\left(T_{1}^{R}\right)^{-1}=b_{n-1}, \quad(n>1 / 2), \\
T_{1}^{R} d_{n}\left(T_{1}^{R}\right)^{-1}=d_{n+1}, \\
T_{1}^{R} b_{1 / 2}\left(T_{1}^{R}\right)^{-1}=d_{1 / 2}^{\dagger},
\end{gathered}
$$

while $T_{1}^{L}$ gives rise to a spectral flow for the left-handed particles,

$$
\begin{gathered}
T_{1}^{L} \delta_{n}\left(T_{1}^{L}\right)^{-1}=\delta_{n-1}, \quad(n>1 / 2), \\
T_{1}^{L} \beta_{n}\left(T_{1}^{L}\right)^{-1}=\beta_{n+1}, \\
T_{1}^{L} \delta_{1 / 2}\left(T_{1}^{L}\right)^{-1}=\beta_{1 / 2}^{\dagger} .
\end{gathered}
$$

The action of $R$ is analogous to charge conjugation,

$$
R b_{n} R^{-1}=-d_{n},
$$




$$
R \beta_{n} R^{-1}=-\delta_{n}
$$

The $a_{N}$ and $\alpha_{N}$ are invariant under both $T_{1}^{R / L}$ and $R$. From the behavior of the Fock operators it is straightforward to deduce the behavior of the elements of the Kac-Moody algebra under $T_{1}^{R / L}$ and $R$, and show that the algebra is invariant.

It is also convenient to introduce a set of states $\left|V_{M}\right\rangle$, where $M$ is any integer, which are related to one another by $T_{N} \equiv T_{N}^{R} T_{N}^{L}$ transformations. The Fock vacuum $|0\rangle$ is defined to be $\left|V_{0}\right\rangle$, and

$$
\left|V_{M}\right\rangle \equiv\left(T_{1}\right)^{M}\left|V_{0}\right\rangle,
$$

with $\left(T_{1}\right)^{-1}=T_{-1}$. It is straightforward to determine the particle content of the $\left|V_{M}\right\rangle$ from the properties of the $T_{1}$ transformation [28]. One finds

$$
\begin{aligned}
& \left|V_{N}\right\rangle=d_{N-1 / 2}^{\dagger} \beta_{N-1 / 2}^{\dagger} \cdots d_{1 / 2}^{\dagger} \beta_{1 / 2}^{\dagger}|0\rangle, \\
& \left|V_{-N}\right\rangle=\delta_{N-1 / 2}^{\dagger} b_{N-1 / 2}^{\dagger} \cdots \delta_{1 / 2}^{\dagger} b_{1 / 2}^{\dagger}|0\rangle,
\end{aligned}
$$

for $N \geqslant 0$. These states are thus analogous to the " $n$ vacua" (2.46) and (2.47) found in the Schwinger model.

The operator $R$ satisfies $R^{2}=1$, so that its action on the Fock vacuum may be defined to be

$$
R|0\rangle= \pm|0\rangle .
$$

Along with Eqs. (4.12) and (4.13), this choice defines the action of $R$ on all states. Without loss of generality we may take the plus sign in Eq. (4.17). Then $R$ interchanges $\left|V_{N}\right\rangle$ and $\left|V_{-N}\right\rangle$ :

$$
R\left|V_{N}\right\rangle=(-1)^{N}\left|V_{-N}\right\rangle .
$$

The factor $(-1)^{N}$ arises from the different ordering of the left- and right-moving creation operators in Eqs. (4.15) and (4.16).

\section{EQUATIONS OF MOTION}

It is straightforward to derive the equations of motion for the theory in the gauge we have chosen. In the color helicity basis the Dirac equation separates into

$$
\begin{gathered}
\partial_{-} \psi_{L}=0, \\
\partial_{-} \phi_{L}=-i g v \phi_{L}, \\
\partial_{+} \psi_{R}=i g\left[A_{-} \phi_{R}-A_{+} \phi_{R}^{\dagger}\right], \\
\partial_{+} \phi_{R}=i g\left[A_{+} \psi_{R}-A_{3} \phi_{R}\right] .
\end{gathered}
$$

In addition we have Gauss' law,

$$
\begin{gathered}
-\partial_{-}^{2} A_{3}=g J_{3}^{R}, \\
-\left(\partial_{-} \pm i g v\right)^{2} A_{ \pm}=g J_{ \pm}^{R},
\end{gathered}
$$

and Ampere's law,

$$
\partial_{+} \partial_{-} A_{3}+i g\left[A_{+}\left(\partial_{-}-i g v\right) A_{-}-A_{-}\left(\partial_{-}+i g v\right) A_{+}\right]=g J_{3}^{-},
$$

$\partial_{+}\left[\left(\partial_{-} \pm i g v\right) A_{ \pm}\right]$

$$
\pm i g\left[A_{3}\left(\partial_{-} \pm i g v\right) A_{ \pm}-A_{ \pm} \partial_{-} A_{3}\right]=g J_{ \pm}^{-} .
$$

These latter relations will require particularly careful consideration, as they explicitly connect left- and right-handed quantities. In particular we will find (as in the Schwinger model) that some of these equations can only be satisfied in a subspace of the full Hilbert space of the theory. This subspace will be defined to be the physical one.

The current operators appearing in Eqs. (5.5)-(5.8) include the gauge corrections computed in Eq. (3.21). Because of the gauge choice and the twisted boundary conditions, however, the corrections to the color \pm components of $J^{R}$ vanish.

We shall discuss the implementation of the Dirac equation and Ampere's law below; for the moment let us consider the solution of Eqs. (5.5) and (5.6). As is usual in light-front field theory, these relations mainly serve to determine the field $A$ on $x^{+}=0$ in terms of the dynamical degrees of freedom. Note that in the gauge we have chosen, Gauss' law separates and its individual components can be solved directly; this is the motivation for introducing the color helicity basis.

Equation (5.5) can be solved immediately to obtain the normal mode part of $A_{3}$ on $x^{+}=0$ :

$$
A_{3}\left(0, x^{-}\right)=\frac{g L}{2 \pi^{2}} \sum_{N=-\infty}^{\infty}, \frac{C_{N}^{3}}{N^{2}} e^{-i N \pi x^{-} / L} .
$$

The zero mode of $A_{3}$ is not determined by Eq. (5.5). We shall return to this problem in a moment, but for now note that since there is no zero mode on the left-hand side of Eq. (5.5), the zero mode of the right-hand side must also vanish. This gives

$$
0=C_{0}^{3}-z_{R}
$$

This relation determines $z_{R}$ in terms of a charge operator, subject to the same caveat we had in the Schwinger model: it is inconsistent to take $z_{R}$ to be an operator involving rightmoving fermions. We shall therefore rewrite Eq. (5.10) in the form

$$
0=C_{0}^{3}+D_{0}^{3}-D_{0}^{3}-z_{R}
$$

and take

$$
z_{R}=-D_{0}^{3}
$$

What remains of the three-color component of Gauss' law is then

$$
C_{0}^{3}+D_{0}^{3}=0
$$

which can be imposed as an eigenvalue condition defining physical states.

Now let us consider Eq. (5.6). Inserting the operator solution (5.12) for $z_{R}$ this becomes

$$
-\left(\partial_{-} \mp \frac{i \pi}{L} D_{0}^{3}\right)^{2} A_{ \pm}=g J_{ \pm}^{R} .
$$


The choice of twisted boundary conditions results in the covariant derivatives having no zero eigenvalues, so they can be inverted to give

$$
A_{ \pm}\left(0, x^{-}\right)=\frac{g L}{2 \pi^{2}} \sum_{n=-\infty}^{\infty} \frac{C_{n}^{\mp}}{\left(n \pm D_{0}^{3}\right)^{2}} e^{-i n \pi x^{-} / L} .
$$

\section{POINCARÉ GENERATORS}

As discussed previously, the Poincare generators $P^{ \pm}$receive contributions from both parts of the initial-value surface [Eq. (2.10)]. We shall denote the contributions coming from integrating over $x^{+}=0$ and $x^{-}=0$ by $P_{R}^{ \pm}$and $P_{L}^{ \pm}$, respectively.

It is straightforward to work out the energy-momentum tensor following the usual Noether procedure. We obtain (with $\partial_{-} A^{+}=0$ )

$$
\begin{aligned}
& \Theta^{+-}=-2 \operatorname{Tr}\left(F^{+-} \partial_{+} A^{+}\right)-\operatorname{Tr}\left(F^{+-} F^{+-}\right) \\
&+\frac{i}{\sqrt{2}} \operatorname{Tr}\left[\Psi_{R} \partial^{-} \Psi_{R}-\left(\partial^{-} \Psi_{R}\right) \Psi_{R}\right] \\
& \Theta^{-+}=-2 \operatorname{Tr}\left(F^{+-} \partial_{-} A^{-}\right)-\operatorname{Tr}\left(F^{+-} F^{+-}\right) \\
&+\frac{i}{\sqrt{2}} \operatorname{Tr}\left[\Psi_{L} \partial^{+} \Psi_{L}-\left(\partial^{+} \Psi_{L}\right) \Psi_{L}\right] \\
& \Theta^{++}= \frac{i}{\sqrt{2}} \operatorname{Tr}\left[\Psi_{R} \partial^{+} \Psi_{R}-\left(\partial^{+} \Psi_{R}\right) \Psi_{R}\right] \\
& \Theta^{--}=\frac{i}{\sqrt{2}} \operatorname{Tr}\left[\Psi_{L} \partial^{-} \Psi_{L}-\left(\partial^{-} \Psi_{L}\right) \Psi_{L}\right]-2 \operatorname{Tr}\left(F^{-+} \partial_{+} A^{-}\right)
\end{aligned}
$$

As in the Schwinger model, these lead to expressions we cannot evaluate: they involve integrals of fields on surfaces where we do not know them. We will follow the rule discussed previously, and simply drop the terms we do not know how to calculate. In the end we shall justify our results by showing that they correctly translate all fields and satisfy the Poincare algebra.

First let us construct $P^{+}$. Using the rule of dropping terms we do not know how to calculate, we obtain

$$
P^{+}=i \sqrt{2} \int_{0}^{2 L} d x^{-} \operatorname{Tr}\left(\Psi_{R} \partial_{-} \Psi_{R}\right)
$$

The operator product in this expression is singular and requires regularization and renormalization. This is accomplished as before by splitting the product in $x^{-}$, introducing an appropriate exponential factor to maintain gauge invariance. We find

$$
P^{+}=\sum_{N>0}\left(\frac{N \pi}{L}\right) a_{N}^{\dagger} a_{N}+\sum_{n>0}\left(\frac{n \pi}{L}\right)\left[b_{n}^{\dagger} b_{n}+d_{n}^{\dagger} d_{n}\right]-\frac{\pi}{2 L} z_{R}^{2},
$$

which leads to

$$
\begin{aligned}
P^{+}= & \sum_{N>0}\left(\frac{N \pi}{L}\right) a_{N}^{\dagger} a_{N}+\sum_{n>0}\left(\frac{n \pi}{L}\right)\left[b_{n}^{\dagger} b_{n}+d_{n}^{\dagger} d_{n}\right] \\
& -\frac{\pi}{2 L}\left(D_{0}^{3}\right)^{2}
\end{aligned}
$$

when Eq. (5.12) is used. This expression will be tested further for consistency below and found to be satisfactory.

Next let us discuss $P^{-}$. The left-moving contribution is given by

$$
P_{L}^{-}=i \sqrt{2} \int_{0}^{2 L} d x^{+} \operatorname{Tr}\left(\Psi_{L} \partial_{+} \Psi_{L}\right)
$$

This operator product is singular, and is regulated by a gauge-corrected splitting in $x^{+}$. We find

$$
\begin{aligned}
P_{L}^{-}= & \sum_{N>0}\left(\frac{N \pi}{L}\right) \alpha_{N}^{\dagger} \alpha_{N}+\sum_{n>0}\left(\frac{n \pi}{L}\right)\left[\beta_{n}^{\dagger} \beta_{n}+\delta_{n}^{\dagger} \delta_{n}\right] \\
& -\frac{g^{2}}{2 \pi} \int_{0}^{2 L} d x^{+} \operatorname{Tr}\left(A^{2}\right) .
\end{aligned}
$$

Again, we do not know most of $A$ on the surface $x^{-}=0$ so we keep only the part we do know on that surface. This will turn out to be the zero mode of $A_{3}$, which will be shown to be $x^{+}$independent. Thus we have

$$
P_{L}^{-}=\sum_{N>0}\left(\frac{N \pi}{L}\right) \alpha_{N}^{\dagger} \alpha_{N}+\sum_{n>0}\left(\frac{n \pi}{L}\right)\left[\beta_{n}^{\dagger} \beta_{n}+\delta_{n}^{\dagger} \delta_{n}\right]-\frac{\pi}{2 L} z_{L}^{2} .
$$

The contribution from the surface $x^{+}=0$ has the standard form one expects, in $(1+1)$-dimensional YM theory coupled to matter,

$$
P_{R}^{-}=-g^{2} \int_{0}^{2 L} d x^{-} \operatorname{Tr}\left(J^{+} \frac{1}{D_{-}^{2}} J^{+}\right) .
$$

None of these operator products are singular so evaluating this expression is straightforward. The result is most elegantly expressed in terms of the $C$ 's. We find

$$
\begin{aligned}
P_{R}^{-}= & \frac{g^{2} L}{4 \pi^{2}}\left[\sum_{N=-\infty}^{\infty} \frac{1}{N^{2}} C_{N}^{3} C_{-N}^{3}\right. \\
& \left.+\sum_{n=-\infty}^{\infty} \frac{1}{\left(n-D_{0}^{3}\right)^{2}}\left\{C_{n}^{+}, C_{-n}^{-}\right\}\right] .
\end{aligned}
$$

There is a problem with this expression, however. The presence of $D_{0}^{3}$ in $P_{R}^{-}$is in conflict with the (kinematical) Heisenberg equation for $\phi_{L}$. We should have

$$
-i\left[\phi_{L}\left(x^{+}, 0\right), P^{-}\right]=\partial_{+} \phi_{L}\left(x^{+}, 0\right),
$$

which is already accomplished by the free part of $P_{L}^{-}$. Since $\left[\phi_{L}, D_{0}^{3}\right]=\phi_{L}$ the interaction terms in $P_{R}^{-}$spoil Eq. (6.13). To cure this problem we can simply modify $P_{R}^{-}$by replacing $D_{0}^{3}$ with an operator that is equal to it in the physical subspace. The natural thing to try is the substitution $D_{0}^{3} \rightarrow-C_{0}^{3}$, motivated by Eq. (5.13). This is potentially in 
conflict with the Dirac equation for $\phi_{R}$, however. Before the substitution we obtained the correct commutator of $\phi_{R}$ with $P^{-}$, but afterwards the fact that $\left[\phi_{R}, C_{0}^{3}\right] \neq 0$ leads to a new and unwanted term in $\left[\phi_{R}, P^{-}\right]$:

$$
\left[\phi_{R}, P^{-}\right]=\frac{g^{2} L}{4 \pi^{2}} \sum_{n}\left[\phi_{R}, \frac{1}{\left(n+C_{0}^{3}\right)^{2}}\right]\left\{C_{n}^{+}, C_{-n}^{-}\right\}+\cdots,
$$

where the dots represent the terms we had originally. Now this extra term can be shown to vanish in matrix elements between states satisfying Eq. (5.13), but this happens for a fairly trivial reason: it is a colored operator and the physical states are required to be colorless. A less trivial check is to consider the commutator of a colorless operator such as $\phi_{R}^{\dagger} \phi_{R}$ with our new $P^{-}$. In fact the commutator of this operator also reduces, in the subspace defined by Eq. (5.13), to what we obtain from the Dirac equation, so the modified $P^{-}$appears to be consistent. Presumably one wants all relations derivable from the equations of motion to be recovered in the physical subspace. It would be helpful to have a more detailed understanding of this point, as well as the extent to which Eq. (6.12) satisfies the necessary conditions.

Equations (6.7), (6.10), and (6.12), with $D_{0}^{3} \rightarrow-C_{0}^{3}$, are our trial forms for $P^{ \pm}$. Our next task is to check whether these correctly reproduce the Dirac equation and Ampere's law for this theory. This is a straightforward exercise in commuting fields with $P^{ \pm}$and comparing the results with the corresponding equations of motion.

It turns out that the Dirac equation for $\psi_{R}$ is satisfied if

$$
z_{L}=-C_{0}^{3},
$$

which we shall take to be a strong (operator) equality. This determines the zero mode of $A_{3}$, which we were not able to fix using Gauss' law. Note that $z_{L}$ commutes with $P^{-}$, and so is $x^{+}$independent as promised.

Next, Ampere's law is satisfied if

$$
D_{N}^{3}=D_{n}^{ \pm}=0 \text {. }
$$

These conditions must be realized weakly, in matrix elements between states. We shall require physical states to satisfy

$$
D_{N}^{3}|\Phi\rangle=0 \quad(N>0),
$$

in analogy with the Schwinger model. Note, however, that because

$$
\left[\left(C_{0}^{3}+D_{0}^{3}\right), D_{n}^{ \pm}\right]= \pm D_{n}^{ \pm}
$$

[see Eq. (3.35)], matrix elements of $D_{n}^{ \pm}$between states that satisfy Eq. (5.13) are automatically zero. It is therefore not necessary to impose the condition $D_{n}^{ \pm} \approx 0$ separately.

Finally, the zero mode of the color 3 component of Ampere's law reduces to

$$
\mathcal{P} \equiv \sum_{n} \frac{1}{\left(C_{0}^{3}+n\right)^{3}}\left\{C_{n}^{+}, C_{-n}^{-}\right\}=0
$$

which again must be realized in matrix elements between physical states. To see that Eq. (6.19) is in fact satisfied in the physical subspace, let us discuss the physical states in more detail. These will be obtained by acting with gaugeinvariant operators built from the right-handed fields on the physical vacuum state $|\Omega\rangle:^{3}$

$$
|\Phi\rangle=\mathcal{O}|\Omega\rangle \text {. }
$$

Now, the transformation $R$ is a symmetry of the theory, as is easily verified from the expressions for $P^{ \pm}$. Without loss of generality we may choose the vacuum to be an eigenstate of $R$ with eigenvalue +1 . Since a gauge-invariant operator is in particular invariant under $R$,

$$
R \mathcal{O} R=\mathcal{O},
$$

it follows that all physical states are also eigenstates of $R$ with eigenvalue +1 . But $\mathcal{P}$ is odd under $R$,

$$
R \mathcal{P} R=-\mathcal{P},
$$

and therefore matrix elements of $\mathcal{P}$ between physical states are zero as required.

The final result is that it appears to be consistent to take $P^{+}$as given in Eq. (6.7) and

$$
\begin{aligned}
P^{-}= & \sum_{N>0}\left(\frac{N \pi}{L}\right) \alpha_{N}^{\dagger} \alpha_{N}+\sum_{n>0}\left(\frac{n \pi}{L}\right)\left[\beta_{n}^{\dagger} \beta_{n}+\delta_{n}^{\dagger} \delta_{n}\right] \\
& -\frac{\pi}{2 L}\left(C_{0}^{3}\right)^{2}+\frac{g^{2} L}{4 \pi^{2}}\left[\sum_{N=-\infty}^{\infty} \frac{1}{N^{2}} C_{N}^{3} C_{-N}^{3}\right. \\
& \left.+\sum_{n=-\infty}^{\infty} \frac{1}{\left(n+C_{0}^{3}\right)^{2}}\left\{C_{n}^{+}, C_{-n}^{-}\right\}\right] .
\end{aligned}
$$

Physical states have vanishing color 3 charge,

$$
\left(C_{0}^{3}+D_{0}^{3}\right)|\Phi\rangle=0,
$$

and satisfy Eq. (6.17). One can check that the operators that annihilate physical states commute with $P^{+}$and $P^{-}$, so the physical subspace is stable. It can also be shown that $\left[P^{+}, P^{-}\right]=0$, as required.

\section{AXIAL ANOMALY}

As a further check of the formulation, let us now discuss the axial anomaly in this theory. We shall focus on the color 3 part of the currents, for which the anomaly relation reads

$$
\partial_{\mu} J_{5}^{\mu, 3}=\frac{g}{2 \pi} \epsilon^{\mu \nu} F_{\mu \nu}^{3} .
$$

In $1+1$ dimensions, the axial current $J_{5}^{\mu}=-\left[\Psi, \gamma^{\mu} \gamma^{5} \Psi\right] / \sqrt{2}$ is related to the vector current $J^{\mu}$ through $J_{5}^{\mu}=\left(J^{R},-J^{L}\right)$. In addition, it can be shown that

\footnotetext{
${ }^{3}$ Conditions (6.17) remove from the physical subspace any states with a left-handed particle content beyond what is present in the vacuum.
} 
covariant derivatives reduce to partial derivatives for $J^{\mu, 3}$ so the conservation equations below take the Abelian form for the matter currents.

To check Eq. (7.1) we first calculate $\partial_{+} J_{3}^{R}$ from

$$
i\left[P^{-}, J_{3}^{R}(x)\right]=\partial_{+} J_{3}^{R}(x) .
$$

Using

$$
\left[\widetilde{J}_{3}^{R}\left(0, x^{-}\right), \widetilde{J}_{3}^{R}\left(0, y^{-}\right)\right]=\frac{i}{2 \pi} \delta^{\prime}\left(x^{-}-y^{-}\right)
$$

we find

$$
\partial_{+} J_{3}^{R}\left(0, x^{-}\right)=\frac{g}{2 \pi} \partial_{-} A_{3}\left(0, x^{-}\right),
$$

where we have chosen to evaluate the currents at $\left(0, x^{-}\right)$. Similarly, we can compute $\partial_{-} J_{3}^{L}$ by commuting it with $P^{+}$. Since $\widetilde{J}_{3}^{L}$ is independent of $x^{-}$by the equations of motion, the only contribution comes from the gauge correction to $J_{3}^{L}$. Thus we find

$$
\partial_{-} J_{3}^{L}\left(0, x^{-}\right)=-\frac{g}{2 \pi} \partial_{-} A_{3}\left(0, x^{-}\right) .
$$

Combining these results we then obtain

$$
\begin{aligned}
\partial_{\mu} J_{3}^{\mu} & =\partial_{+} J_{3}^{R}+\partial_{-} J_{3}^{L} \\
& =0
\end{aligned}
$$

and

$$
\begin{aligned}
\partial_{\mu} J_{3}^{5, \mu} & =\partial_{+} J_{3}^{R}-\partial_{-} J_{3}^{L}=\frac{g}{\pi} \partial_{-} A_{3} \\
& =\frac{g}{2 \pi} \epsilon^{\mu \nu} F_{\mu \nu}^{3},
\end{aligned}
$$

as expected. The formulation of the theory therefore appears to be satisfactory, and we can now study the structure of the ground state.

\section{VACUUM STATES}

It is straightforward to verify that the states $\left|V_{N}\right\rangle$ are all degenerate (they have $P^{+}=P^{-}=0$ ) and lie in the physical subspace. The physical vacuum states will thus be appropriate superpositions of these, constructed to be (phase) invariant under the residual $T_{1}$ and $R$ symmetries.

The most general superposition consistent with $T_{1}$ invariance is simply

$$
|\theta\rangle=\sum_{N=-\infty}^{\infty} e^{-i N \theta}\left|V_{N}\right\rangle,
$$

which satisfies

$$
T_{1}|\theta\rangle=e^{i \theta}|\theta\rangle .
$$

Acting with the Weyl transformation $R$ then gives

$$
\begin{aligned}
R|\theta\rangle & =\sum_{N=-\infty}^{\infty} e^{-i N \theta}(-1)^{N}\left|V_{-N}\right\rangle \\
& =\sum_{N=-\infty}^{\infty} e^{i N(\theta-\pi)}\left|V_{N}\right\rangle .
\end{aligned}
$$

This is equal to $|\theta\rangle$ up to a phase only for $\theta= \pm \pi / 2$. We therefore have a pair of distinct physical vacuum states labeled by a discrete vacuum angle. We shall refer to these physical vacua as $\left|\Omega_{ \pm}\right\rangle$.

We have here motivated the formation of the superposition (8.1) as a way of resolving the residual (large) gauge invariance of the theory. It is presumably also necessary to build the theory on a vacuum of this form in order to satisfy the cluster property, as in the Schwinger model. To verify this explicitly for the present model, however, we would need to do a more complete dynamical calculation.

Let us now consider whether this vacuum structure has any affect on the spectrum of the theory, that is, whether the spectrum depends on the vacuum angle $\theta= \pm \pi / 2$. Consider calculating a matrix element of $P^{-}$between any two physical states:

$$
\left\langle\Omega_{ \pm}\left|\mathcal{O}^{\prime}\left(P_{R}^{-}+P_{L}^{-}\right) \mathcal{O}\right| \Omega_{ \pm}\right\rangle,
$$

where $\mathcal{O}$ and $\mathcal{O}^{\prime}$ are gauge-invariant operators constructed from the right-handed fields. Since $P_{L}^{-}$commutes with these it simply passes through to act on the vacuum, where it gives zero. In addition, since $\mathcal{O}$ and $\mathcal{O}^{\prime}$ contain no left-handed fields, the left-handed particles in the vacuum serve to "diagonalize" the matrix element between the different $\left|V_{M}\right\rangle$ :

$$
\left\langle\Omega_{ \pm}\left|\mathcal{O}^{\prime} P_{R}^{-} \mathcal{O}\right| \Omega_{ \pm}\right\rangle=\sum_{N=-\infty}^{\infty}\left\langle V_{N}\left|\mathcal{O}^{\prime} P_{R}^{-} \mathcal{O}\right| V_{N}\right\rangle .
$$

Now $\mathcal{O}$ and $\mathcal{O}^{\prime}$ are invariant under $T_{1}$, and furthermore it can be shown that

$$
T_{1} P_{R}^{-} T_{1}^{-1}=P_{R}^{-} .
$$

Inserting factors of $\left(T_{1}^{-1} T_{1}\right)^{N}$ between the states and operators we therefore find

$$
\left\langle V_{N}\left|\mathcal{O}^{\prime} P_{R}^{-} \mathcal{O}\right| V_{N}\right\rangle=\left\langle V_{0}\left|\mathcal{O}^{\prime} P_{R}^{-} \mathcal{O}\right| V_{0}\right\rangle .
$$

All the matrix elements on the right-hand side of Eq. (8.5) are thus identical, and so

$$
\left\langle\Omega_{ \pm}\left|\mathcal{O}^{\prime} P_{R}^{-} \mathcal{O}\right| \Omega_{ \pm}\right\rangle=\left\langle V_{0}\left|\mathcal{O}^{\prime} P_{R}^{-} \mathcal{O}\right| V_{0}\right\rangle,
$$

up to the (infinite) normalization factor necessary for the state $\left|\Omega_{ \pm}\right\rangle$. Finally, we note that $C_{0}^{3}\left|V_{0}\right\rangle=0$, so that we may replace $C_{0}^{3}$ by zero in the expression for $P_{R}^{-}$. The resulting $P_{R}^{-}$is simply the usual discrete light-cone quantized (DLCQ) Hamiltonian for this theory, that is, with the zero modes discarded. The final result is that matrix elements of the Hamiltonian in the full theory are equal to those we would obtain by taking the trivial Fock vacuum $\left|V_{0}\right\rangle$ and ignoring the zero modes. Thus the standard DLCQ procedure gives the correct spectrum for this theory. 
We should perhaps emphasize that in more complicated theories, such as QCD, the analogous result will certainly not hold. In that case there are physical quantities that do depend on the vacuum angle $\theta$, and it will not be possible to correctly reproduce these by neglecting the vacuum structure.

\section{THE CONDENSATE}

It is generally believed that $\mathrm{YM}_{1+1}$ coupled to adjoint fermions develops a condensate $\Sigma \equiv\langle\Omega|\operatorname{Tr}(\bar{\Psi} \Psi)| \Omega\rangle$. Thus far $\Sigma$ has been calculated in the large- $N$ limit [29] and in the small-volume limit for $\mathrm{SU}(2)$ using equal-time quantization [30]. A condensate was also computed in a chiral version of this theory containing only right-handed fermions [28]. In that calculation it was the field itself which acquired a vacuum expectation value and the result was fundamentally different from what we are considering here.

It is straightforward to compute $\Sigma$ in the vacuum (8.1). To be specific, we shall evaluate $\Sigma$ at the spacetime point $\left(0, x^{-}\right)$. The terms in $\operatorname{Tr}(\bar{\Psi} \Psi)$ that can contribute to the vacuum expectation value are

$$
\operatorname{Tr}(\bar{\Psi} \Psi)=i\left(\phi_{L}^{\dagger} \phi_{R}-\phi_{R}^{\dagger} \phi_{L}\right)+\cdots .
$$

These operator products are not singular, so point splitting is not required.

The field $\phi_{R}\left(0, x^{-}\right)$is just given by its initial value (3.9), of course. To obtain $\phi_{L}$ at $\left(0, x^{-}\right)$we must solve its equation of motion, i.e., Eq. (5.2), with the noncommuting factors on the RHS symmetrized:

$$
\partial_{-} \phi_{L}=\frac{i \pi}{2 L}\left(D_{0}^{3} \phi_{L}+\phi_{L} D_{0}^{3}\right) .
$$

Integrating this gives $\phi_{L}\left(0, x^{-}\right)$in terms of its initial value at the corner point $(0,0)$ :

$$
\phi_{L}\left(0, x^{-}\right)=e^{i \pi x^{-} D_{0}^{3} / 2 L} \phi_{L}(0,0) e^{i \pi x^{-} D_{0}^{3} / 2 L} .
$$

With these results it is straightforward to evaluate the condensate; we find

$$
\left\langle\Omega_{ \pm}|\operatorname{Tr}(\bar{\Psi} \Psi)| \Omega_{ \pm}\right\rangle= \pm \frac{1}{\sqrt{2} L} .
$$

Note that the result is independent of $x^{-}$, as it should be. This happens because the exponential factors in Eq. (9.3) acting on the $\left|V_{N}\right\rangle$ exactly compensate the exponentials from the field expansion (3.9). ${ }^{4}$ In addition, however, the condensate is proportional to $1 / L$, and so vanishes in the continuum limit.

This type of behavior is also found in the light-front version of the Schwinger model discussed in [11]. In that case the $1 / L$ behavior can be traced to the crude treatment of the small- $p^{+}$region-in particular the violation of parity-that results when periodicity conditions are imposed on null planes [31]. In the Schwinger model the problem can be

\footnotetext{
${ }^{4}$ Note that use of the properly symmetrized solution (9.3) is crucial for this to work.
}

cured by forcibly parity symmetrizing the theory after it has been solved. In the present example this sort of approach may not be practical, since the model is not analytically soluble.

As discussed previously, the condensate, or more precisely the presence of nontrivial vacuum structure, has no effect on the mass spectrum of the theory. This result is in accordance with recent work of Kutasov and Schwimmer [16], who claim that there are classes of two-dimensional YM theories which have the same massive spectrum. A necessary condition for this universality is the decoupling of the massless (vacuum) and massive sectors. Our construction exhibits this directly. In particular, the massive spectrum may be obtained by neglecting the vacuum structure and gauge field zero modes, that is, by applying the naive light-front formalism. In addition, the only left-moving quanta that enter physical states reside in the vacuum; the physical excitations are built entirely from the right movers.

It would be interesting to study whether the spectrum depends on the vacuum angle when a fermion mass is turned on, as, for example, occurs in the massive Schwinger model. At present we have nothing definite to say on this question, although the condensate $\Sigma$ does arise in at least one interesting context in the massive theory. It has been shown recently that two-dimensional gauge theories with massless fermions can screen "fractional" test charges - charges in representations of the gauge group that are smaller than the one carried by the dynamical fermions in the theory [32]. Thus, for example, in the theory with adjoint fermions the fundamental Wilson loop exhibits a perimeter-law behavior. When the dynamical fermions are given a mass $m$, however, the screening disappears and fractional charges are confined, with a string tension

$$
\sigma=2 m \Sigma
$$

It is unclear whether this has implications for the question of whether the spectrum of the theory with massive fermions itself is affected by the condensate [32].

\section{CONCLUSIONS}

We have shown that the $Z_{2}$ vacuum structure of SU(2) gauge theory coupled to adjoint fermions in $1+1$ dimensions can in fact be successfully reproduced in the light-front framework. We have found a pair of (degenerate) physical vacuum states, and a nonzero "chiral" condensate which is sensitive to the vacuum physics. This vacuum structure decouples from the massive spectrum, however, consistent with Ref. [16].

Three ingredients are essential for obtaining this structure. First, it is necessary to include a complete set of independent degrees of freedom, that is, independent fields initialized on two different null planes. The surface $x^{+}=0$ does not define a Cauchy problem for the left-moving degrees of freedom. For some purposes it may be possible to ignore this subtlety. In the present case, for example, the physics of massive states can be correctly recovered by ignoring the left movers and zero modes. This is a feature peculiar to $(1+1)$ dimensional gauge theories with massless matter, however [16]. It is not expected to be true in more complicated theo- 
ries like $\mathrm{QCD}$, where there is a strong coupling between massless and massive states.

Second, it is important to pay close attention to the interplay between the gauge choice and boundary conditions. Given the periodicity conditions we have imposed to regulate the theory, it is not possible to gauge all the zero modes of $A_{\mu}$ to zero. Certain of these must be retained in the theory and their properties determined. This situation is quite familiar even in equal-time quantization, when one regulates with equal-time periodicity conditions and attempts to impose a spacelike axial gauge (see, e.g., [33,34]).

Finally, it is necessary to carefully define singular operator products in a gauge-invariant way. The resulting gauge corrections to $P^{ \pm}$are what allow certain states which contain pairs of right- and left-moving quanta to actually be degenerate with the bare vacuum. Furthermore, the gauge corrections to the current operators are crucial for determining the gauge field zero modes, as well as for obtaining the correct anomaly relation.

The vacuum states have a much simpler structure in the light-front representation than at equal time. (This is also true in the Schwinger model [11].) However, the formulation with periodicity conditions on the characteristic surfaces has the property that some of the details of the operator products, such as the condensate, do not approach their continuum values as the periodicity length is taken to infinity. The degree to which one may lose the ability to represent some aspects of the physics in the discretized light-front approach is not entirely understood. It would be very interesting to have a more general understanding of this point.

It would also be of interest to extend this sort of construction to the case of massive fermions, where the vacuum structure can play a more meaningful physical role. In the massive Schwinger model, for example, the spectrum of states depends on the vacuum angle $\theta$, a property shared by QCD.

Finally, it will not have escaped the reader that the construction we have presented relies to an uncomfortable degree on trial and error. We know of no standard procedure, analogous to the textbook treatment for equal-time field theory, which leads directly to the correct dynamical operators. Instead, we start from the canonical expressions for $P^{ \pm}$, discarding terms we do not know how to evaluate and including gauge corrections arising from the renormalization of singular operator products. Further modifications may be necessary in response to checks of consistency, in particular the replacement of troublesome operators with operators to which they are weakly equivalent, i.e., equal in the physical subspace. Of course, the definition of the physical subspace, and hence which operators may be considered to be weakly equivalent, itself depends on the form of the Poincare generators: these determine which Heisenberg equations are not obtained directly as operator relations, and thereby fix the conditions which must be satisfied by states in order for these to hold in a weak sense. The procedure thus has an unpleasantly circular character. It would be of great interest to have a more straightforward formulation of light-front field theory, particularly as the addition of further spacetime dimensions, and the associated renormalization problems, can only increase the difficulties.

\section{ACKNOWLEDGMENTS}

It is a pleasure to thank David Kutasov, Pierre van Baal, and Ariel Zhitnitsky for helpful discussions. S.S.P. would also like to acknowledge the hospitality of the Aspen Center for Physics, where part of this work was completed. This work was supported in part by grants from the U.S. Department of Energy.
[1] P. A. M. Dirac, Rev. Mod. Phys. 21, 392 (1949).

[2] Overviews of the light-front approach with references to earlier work may be found in S. J. Brodsky and H.-C. Pauli, in Recent Aspects of Quantum Fields, edited by H. Mitter and $\mathrm{H}$. Gausterer, Lecture Notes in Physics Vol. 396 (SpringerVerlag, Berlin, 1991); S. J. Brodsky, G. McCartor, H.-C. Pauli, and S. S. Pinsky, Part. World 3, 109 (1993); R. J. Perry, in Hadron Physics 94: Topics on the Structure and Interaction of Hadronic Systems, Proceedings of the Workshop, Gramado, Brazil, 1994, edited by V. H. Herscovitz et al. (Workshop Scientific, Singapore, 1995), hep-th/9407056; W.-M. Zhang, Chin. J. Phys. 32, 717 (1994); M. Burkardt, Adv. Nucl. Phys. 23, 1 (1996); S. J. Brodsky and D. G. Robertson, in Confinement Physics, edited by S. D. Bass and P. A. M. Guichon (Editions Frontières, France, 1996); A. Harindranath e-print hep-ph/9612244.

[3] T. Maskawa and K. Yamawaki, Prog. Theor. Phys. 56, 270 (1976).

[4] H.-C. Pauli and S. J. Brodsky, Phys. Rev. D 32, 1993 (1985); 32, 2001 (1985).

[5] T. Heinzl, S. Krusche, S. Simbürger, and E. Werner, Z. Phys. C 56, 415 (1992).
[6] D. G. Robertson, Phys. Rev. D 47, 2549 (1993).

[7] C. M. Bender, S. Pinsky, and B. van de Sande, Phys. Rev. D 48, 816 (1993).

[8] S. S. Pinsky and B. van de Sande, Phys. Rev. D 49, 2001 (1994).

[9] J. Hiller, S. S. Pinsky, and B. van de Sande, Phys. Rev. D 51, 726 (1995).

[10] A. C. Kalloniatis, H.-C. Pauli, and S. S. Pinsky, Phys. Rev. D 50, 6633 (1994).

[11] G. McCartor, Z. Phys. C 52, 611 (1991); 64, 349 (1994).

[12] A. C. Kalloniatis and D. G. Robertson, Phys. Lett. B 381, 209 (1996).

[13] E. Witten, Nuovo Cimento A 51, 325 (1979).

[14] L. D. Paniak, G. W. Semenoff, and A. R. Zhitnitsky, Nucl. Phys. B487, 191 (1997).

[15] A. Smilga, Phys. Rev. D 49, 6836 (1994).

[16] D. Kutasov and A. Schwimmer, Nucl. Phys. B442, 447 (1995).

[17] S. S. Pinsky, Ohio State University report (unpublished), e-print hep-th/9612073.

[18] J. Schwinger, Phys. Rev. 128, 2425 (1962).

[19] D. G. Robertson, Ohio State University Report No. OHSTPYHEP-T-96-011 (unpublished), e-print hep-th/9603205.

[20] G. McCartor, Z. Phys. C 41, 271 (1988). 
[21] V. A. Franke, Yu. A. Novozhilov, and E. V. Prokhvatilov, Lett. Math. Phys. 5, 239 (1981); 5, 437 (1981); Dynamical Systems and Microphysics (Academic Press, New York, 1982).

[22] Y. Nakawaki, Prog. Theor. Phys. 64, 1828 (1980).

[23] P. Goddard and D. Olive, Int. J. Mod. Phys. A 2, 303 (1986).

[24] G. 't Hooft, Commun. Math. Phys. 81, 267 (1981).

[25] M. A. Shifman and A. V. Smilga, Phys. Rev. D 50, 7659 (1994).

[26] S. S. Pinsky and A. C. Kalloniatis, Phys. Lett. B 365, 225 (1995).

[27] V. N. Gribov, Nucl. Phys. B139, 1 (1978).
[28] S. S. Pinsky and D. G. Robertson, Phys. Lett. B 379, 169 (1996).

[29] I. I. Kogan and A. R. Zhitnitsky, Nucl. Phys. B465, 99 (1996).

[30] F. Lenz, M. Shifman, and M. Thies, Phys. Rev. D 51, 7060 (1995).

[31] G. McCartor, Int. J. Mod. Phys. A 12, 1091 (1997).

[32] D. J. Gross, I. R. Klebanov, A. V. Matytsin, and A. Smilga, Nucl. Phys. B461, 109 (1996).

[33] Y. Nakawaki, Prog. Theor. Phys. 70, 1105 (1983).

[34] N. S. Manton, Ann. Phys. (N.Y.) 159, 220 (1985). 